\title{
The Role of Diffusion Weighted Magnetic Resonance Imaging and Subtraction Magnetic Resonance imaging in Assessing Treatment Response of Hepatocellular Carcinoma After Transarterial Chemoembolization \\ Mohammed S. Hassan, Mohammed G. Abdel-Mutaleb, Elhadi I. Ahmed
}

Department of Radiology, Faculty of Medicine -Ain Shams University

Corresponding author: Elhadi I Ahmed; Mobile: 01027667141; Email: hadiizaldeen@ hotmail.com

\begin{abstract}
Background: Hepatocellular carcinoma (HCC) is one of the most common cancers worldwide, and has a poor prognosis unless treated. Ablative therapies are promising treatment options for patients who are not eligible for surgery. Monitoring tumor response after transarterial chemoemolization (TACE) procedure is an important task in oncologic imaging. Early favorable response indicates effectiveness of therapy, while early treatment failure identification is also critical in patient management. In such cases further re-treatment will be mandatory. Aim of work: The aim of this study is to evaluate the efficiency of both diffusion weighted images and subtracted dynamic MRI technique in the detection of residual/ recurrent disease after TACE ablation of non resectable hepatocellular carcinoma (HCC) lesions. Accurate judgment of tumor viability will help diagnose the need for further treatment sessions. Patients and Methods: This study included 32 patients having 54 HCC lesions that underwent transarterial chemoembolization procedure over a period of 6 months (2017-2018) were followed up 1-1.5 months by dynamic MRI. 12 patients of which underwent a second follow up within 3-4 months. Patients' ages ranged between 59 to 73 years (mean age 53.1); 26 patients were males and 6 were females. All patients had liver cirrhosis related to chronic viral hepatitis. Results: In $1^{\text {ry }}$ response and follow up findings post TACE, where good response was defied as disappearance of any intra tumoral arterial enhancement in treated lesions, residual disease was defined as at least $30 \%$ reduction in the sum of diameters of the viable enhancing lesion in the arterial phase, no response was less than $30 \%$ reduction in the enhancing lesion diameters. Progressive disease was defined as $20 \%$ increase in the sum of diameters of the enhancing lesions in comparison to the target lesion diameter at the start of treatment. Conclusion: MRI is a powerful tool in detection of residual tumor viability, quantifying tumor necrosis and detecting complications after TACE. Imaging protocol should include dynamic study combined with post processing subtraction images for better tissue characterization.
\end{abstract}

Keywords: DW MRI, subtraction MRI, hepatocellular carcinoma, transarterial chemoembolization.

\section{INTRODUCTION}

Hepatocellular carcinoma is the fifth most common cancer worldwide, and its incidence is rising in many countries, including the United States. In 2008 it was estimated that 748,300 new cases were diagnosed ${ }^{(\mathbf{1})}$. The major risk factor for development of hepatocellular carcinoma is cirrhosis, particularly cirrhosis related to chronic viral hepatitis, alcoholic cirrhosis, cirrhosis caused by hemochromatosis, and primary biliary cirrhosis (2).

The main curative treatment for HCC is considered to be surgical resection. However, nonsurgically fit patients can benefit from locoregional ablative therapy ${ }^{(2)}$. Ablative techniques can be classified to either chemical or thermal. Chemical ablation refers to the use of ethanol and acetic acid, whereas thermal ablation is achieved by using heat (radiofrequency ablation, microwave ablation, laser ablation); or cold (cryoablation) ${ }^{(3)}$. All these therapeutic measures end in tumor coagulative necrosis ${ }^{(4)}$.

TACE affects the tumor by selective or super-selective embolization of tumor vessels with chemotherapeutic agents reducing the tumoral blood flow. Embolizing particles prolonged contact of the tumor with the chemotherapeutic agents. Lipiodol is used as a carrier for the chemotherapeutic agents. It slowly reaches the sinusoids, and gets trapped within the tumor that lack Kupffer cells ${ }^{(4)}$.

The assessment of the treatment efficacy is vital in determining whether the chemoembolization has been successful or not and hence aids in guiding future therapy ${ }^{(3)}$. Histological evaluation of locoregional treatment efficiency is neither easy nor reasonable for patients. Using tissue biopsy can only be conclusive when it shows viable malignancy. Therefore, repeated negative biopsies do not exclude the presence of residual tumor. Thus monitoring treatment response is indispensable to radiological imaging to evaluate any viable residual tumor ${ }^{(3)}$.

Multidetector computed tomography (MDCT) and magnetic resonance imaging (MRI) are widely used for post treatment monitoring. Triphasic CT is used as the standard imaging technique to evaluate the therapeutic response. It is done immediately post TACE to ensure the retention of lipiodol by the tumor and repeated 1 
month later to detect any tumoral residue or recurrence $^{(4)}$.

The use of lipidol raises two diagnostic challenges in CT. The first is its defective accumulation by necrotic or residual tumor. The second is hyper attenuating retained lipidol causes beam hardening artefact which degrades the image quality ${ }^{(4)}$.

On the contrary MRI is more efficient than MDCT in the detection of viable residual tumor elements following TACE. Thus, the use of MRI is compulsory during the follow-up ${ }^{(4)}$.

Noncontrast T1 and T2 weighted images provide information on morphological and fluid content changes as well as fibrosis; while dynamic contrast enhancement (DCE) can provide information on perfusion. DCE MRI is sensitive to therapy-related changes in blood volume and vascular permeability that can be associated with tumor angiogenesis ${ }^{(5)}$.

Tumors that are adequately treated by TACE are usually replaced by necrosis associated with reduced interstitial tissue fluid because of the concomitant arterial occlusion. As a result, the MRI findings of successful TACE treatment shows increased signal intensity on T1 weighted images, decreased signal intensity on T2 weighted images, and lack or negligible enhancement on arterial phase images. Reduced T2 signal is a particularly distinctive feature of TACE ${ }^{(5)}$.

Enhancement on DCE-MRI post TACE represents either residual tumoral tissue mixed with necrotic regions or granulation tissue. Differentiation is based on the phase of enhancement. Residual tumoral tissue gives early arterial enhancement denoting unsuccessful TACE treatment; whereas granulation tissue shows delayed uptake ${ }^{(5)}$.

Based on the above, evaluation of tumoral response by maximum size e.g. RECIST or mRECIST were not accurate as some tumors show paradoxical enlargement due to hemorrhage, necrosis or edema. Furthermore FHL post treatment show increased $\mathrm{T} 1$ signal which makes residual tumoral contrast enhancement difficult to differentiate. As a result further tools were utilized as functional DWI-MRI or subtraction DCE-MRI (6). Diffusion-weighted imaging (DWI) provides unique information associated with tumor cellularity and cell membrane integrity. Therefore, it may be sensitive to the changes that occur in the tumor microenvironment following treatment, which can be evaluated quantitatively for the calculation of the apparent diffusion coefficient (ADC) ${ }^{(7)}$. Elevated ADC reflects increased mobility of water, either through loss of membrane integrity or an increase in the proportion of total extracellular fluid with a corresponding decrease in cellular size or number, as seen with necrosis or apoptosis. In contrast, reduced ADC reflects a decrease in free extracellular water, either through an increase in total cellular size or number, as can be seen with tumor progression, fibrosis, or edema. Therefore, ADC has the potential to predict the tumor response to treatment weeks before the dimensional changes appear ${ }^{(5)}$.

Subtraction imaging is a technique whereby an unenhanced T1-weighted sequence is digitally subtracted from the identical sequence performed after gadolinium administration. By performing this operation, any native $\mathrm{T} 1$ signal is removed and the remaining signal on the subtracted images is due only to enhancement. Subtraction can make subtle enhancement within a tumor evident and can remove high T1 signal, present due to coagulation necrosis. It can also help differentiate the smooth indistinct peritumoral enhancement seen in benign posttreatment hyperemia from the discontinuous nodular enhancement of viable tumor. Subtraction can make evaluation of these lesions more straightforward $^{(\mathbf{8})}$.

\section{Aim of work}

The aim of this study is to evaluate the efficiency of both diffusion weighted images and subtracted dynamic MRI technique in the detection of residual/ recurrent disease after transarterial chemoembolization (TACE) ablation of non resectable hepatocellular carcinoma (HCC) lesions. Accurate judgment of tumor viability will help diagnose the need for further treatment sessions.

\section{PATIENTS AND METHODS}

This study included 32 patients having $54 \mathrm{HCC}$ lesions that underwent transarterial chemoembolization procedure over a period of 6 months (2017- 2018) were followed up 1-1.5 months by dynamic MRI. 12 patients of which underwent a second follow up within 3-4 months. The study was approved by the Ethics Board of Ain Shams University.

The patients' ages ranged between 59 to 73 years (mean age 53.1); 26 patients were males and 6 were females. All patients had liver cirrhosis related to chronic viral hepatitis.

All cases had been subjected to the following:

- Full clinical assessment.

- Revision of the patient's laboratory investigations including renal function tests (urea and creatinine).

- Revision of the previous radiological investigations done for the patients.

- Written consent was taken from all patients. 
- Patients were scheduled to undergo MRI within 1 month after embolization and were advised for further follow up 3 months later.

- Exclusion criteria:

- Contraindications to contrast media, e.g. patients with renal failure, patients allergic to contrast media.

- Contraindications to magnetic resonance imaging, e.g. claustrophobia in patients contraindicated for anesthesia, non MR compatible cardiac prosthesis, pace makers, metallic plates.
- Liver tumors other than hepatocellular carcinoma.

- Procedures other than TACE e.g. (RF, microwave ablation)

\section{Inclusion criteria:}

- Cases with HCC lesion undergoing only TACE as therapeutic procedure either by beads or lipidol.

No contraindications to MRI.

MRI protocol:

All cases were performed using Philips1.5Tesla MRI scanner (Achieva).

Table (1): The examination parameters of all the used pre and post contrast MR sequences

\begin{tabular}{|c|c|c|c|c|c|}
\hline Sequence & TR (ms) & TE (ms) & FLIP ANGLE $\left(^{\circ}\right)$ & $\begin{array}{l}\text { Slice } \\
\text { thickness } \\
(\mathbf{m m})\end{array}$ & FOV (mm) \\
\hline T1FEP coronal & 10 & 4.6 & 15 & 3.86 & $200-380$ \\
\hline T1 axial & 10 & 4.6 & 15 & 7 & $300-380$ \\
\hline $\begin{array}{l}\text { T1 in and out } \\
\text { of phase }\end{array}$ & 500 & $\begin{array}{l}\text { TE1 } 2.3 \\
\text { TE2 } 4.6 \\
\end{array}$ & 80 & 10 & $300-380$ \\
\hline T2 & $\begin{array}{c}888 \\
3000 \text { (HEAVY T2) }\end{array}$ & 400 & 90 & 10 & $300-380$ \\
\hline THRIVE & 157 & 4.6 & 10 & 10 & $300-380$ \\
\hline DWI & 1212 & 62 & 90 & 10 & $300-380$ \\
\hline
\end{tabular}

\section{Dynamic study:}

Dynamic study was performed after manual bolus injection of $0.1 \mathrm{mmol} / \mathrm{kg}$ body weight of Gd-DTPA and flushed with $20 \mathrm{ml}$ of sterile saline solution from the antecubital vein.

Dynamic imaging using 3D fat-suppressed T1-weighted gradient echo sequence (THRIVE i.e.T1 high resolution isotropic volume examination). A dynamic series consisted of one pre contrast series followed by four successive post contrast series including early arterial, late arterial, and portal phases with 19-21 seconds intervals

(17 seconds for image acquisition with breathholding and 2-4 seconds for re-breathing).

This is followed by 5-min delayed phase imaging. All patients were imaged in end expiration to limit the risk of image misregistration.

\section{b) Diffusion weighted images:}

Functional DWI was performed using single-shot spin-echo echo-planar imaging during one or more breath holds.

Further detailed imaging parameters were as follows:

- Repetition time/echo time $=2500 / 82 \mathrm{~ms}$;

- Slice thickness/gap = 8/4 mm;

- Bandwidth $1.5 \mathrm{kHz} /$ pixel;

- Partial Fourier factor 6/8;

- Non-selective fat saturation; twice refocused spinecho diffusion weighting to reduce eddy-current induced distortion with $\mathrm{b}$ values of 0,300 and 600 $\left.\mathrm{s} / \mathrm{mm}^{2}\right)$.
- ADC parametric maps were reconstructed from each set of DW images acquired at each slice position.

\section{Analysis of the MR images:}

- Images were sent to the workstation (Phillips Extended MR Workspace) for further image processing.

- Subtraction imaging was then performed which is an automated process available on the workstation, whereby an unenhanced T1- weighted sequence is digitally subtracted from the identical sequence performed after gadolinium administration in early angiographic and late arterial phases.

- By this process, any native T1 signal is removed and the remaining signal on the subtracted images is due solely to enhancement.

- DWI were used to create ADC maps and subsequently $\mathrm{ADC}$ values were measured and recorded.

- The morphological features of each lesion were recorded including number of treated lesions, site, size, margins and signal intensity at $\mathrm{T} 1, \mathrm{~T} 2$ and SPAIR images.

- Assessment for presence of complications, residual or recurrent tumor viability.

\section{Dynamic study analysis:}

- We perform early arterial, late arterial, portal and delayed phases after the single pre-contrast series.

- Pattern of enhancement (through the successive dynamic series) was then studied.

Subtraction Dynamic study analysis: 
Subtraction was performed on available workstations and ADC maps were generated. Further evaluation of the cases was done by two experienced readers looking through the provided sequences

- Pattern of enhancement in the subtraction dynamic imaging was then studied.

\section{DWI:}

- Qualitative assessment for restricted and facilitated areas of the treated lesions, where lesions with high signal or low signal were correlated to ADC maps to exclude T2 shine through effect or T2 black out.

- ADC maps were assessed both qualitatively by recording the signal intensity and quantitatively by measuring ADC values in the areas of restriction that appeared pathologically enhancing in the late arterial images.

\section{ADC measurement:-}

- Pixel-based ADC maps were generated on the workstation. ADC was calculated with linear regression analysis of the function $\mathrm{S}=\mathrm{S} 0 \times \exp (-$ $\mathrm{b} \times \mathrm{ADC}$ ), where $\mathrm{S}$ is the signal intensity after application of the diffusion gradient, and $\mathrm{S} 0$ is the signal intensity at $a b$ value of $0 \mathrm{~s} / \mathrm{mm}^{2}$. The three $b$ values $\left(0,300\right.$, and $\left.600 \mathrm{~s} / \mathrm{mm}^{2}\right)$ were used for ADC calculation.

- Areas of hyperintensity on DWI were correlated to T2 WI to exclude the presence of T2 shine through or blackout effects.

- The restricted areas were correlated to THRIVE images to confirm the presence of enhancement.

- A circular ROI (region of interest) was drawn around the suspected lesions either through contrast enhancement (due to residual/recurrent disease or due to reactive enhancement) or intralesional restriction.

- If the lesions were not properly presented on DWI due to their small size or artefacts, THRIVE image was used where the centre of the lesion was determined by the intersection point of two perpendicular lines extending from the upper and the left border of the images, respectively. With the knowledge of the length of each line, the identical lines could be drawn on the corresponding ADC map. With the reference to the lesion size, circular ROI was placed around to measure the ADC of the corresponding lesion.

- If no high signal or enhancement could be identified, the whole lesion was measured.

\section{Interpretation of the MR image:}

- MR images were interpreted by two readers who are experienced in hepatic imaging. Each reader interpreted the dynamic MRI and subtraction MRI images and compared it to the findings of diffusion weighted images and ADC value was recorded.

- Signal of the embolized zone at T1, T2 and SPAIR WIs was classified as: high, low or heterogeneous.

\section{A. Dynamic study interpretation:}

- Arterial phase enhancement and delayed wash out were recorded suggestive of residual tumoral activity.

- Absence of enhancement in the arterial phase was recorded suggestive of proper tumor embolization.

\section{B. Subtraction Dynamic studies interpretation:}

Similarly the dynamic images were interpreted and recorded findings were confirmed / corrected by the subtraction images to prove that the high signal in the arterial phase is due to enhancement and not due to the original precontrast high $\mathrm{T} 1$ signal of the coagulative necrosis.

Interpretation of the presence of ill-defined perilesional parenchymal enhancement:

- Post interventional reactive change are defined as early phase enhancement beyond the embolized cavity on the surrounding liver parenchyma that persists in the delayed phase.

- Perfusion abnormalities (transient hepatic intensity difference) is ill defined parenchymal enhancement during the arterial phase. This occurs in cases of injury of the portal vein or traumatic arterio-portal shunting.

Well defined enhancement at the margin of the embolization zone which may be either:

- Granulation tissue rim: Persistent or delayed phase enhancement.

- Nodular or hallow enhancement: that suggest tumor recurrence.

Pattern of enhancement impressive for tumor progression according to Chopra et al., 2001) ${ }^{(9)}$ :

- Halo type: Irregular, thick rim of enhancement around the ablation zone.

- Nodular type: Nodular foci of enhancement at the margins of the embolization zone.

- Gross enlargement type: Overall increase in the size of the embolization zone.

We categorize the patients into two groups:

- Resolved group (well ablated): no MRI signs of residual or recurrent viability (regardless the presence of newly developed lesions) by both readers.

- Unresolved group (residual): if there is evidence of residual or recurrent tumor by both readers.

\section{Standard of Reference}

It was difficult to obtain pathologic confirmation in patients who underwent TACE embolization because most of these patients do not undergo surgery. In addition, biopsy may result in sampling error as recurrent lesions are mostly small nodules and a negative sample may be wrongly taken from a necrotic region while there may be still viable areas within the lesion.

In addition not all patients returned for their follow up. 
So well-treated (resolved) lesions were considered by absence of enhancement on both the arterial phase of the dynamic study subtraction dynamic study and the residual tumor activity (unresolved) lesions were suggested by:

Presence of enhancement on arterial phase and wash out of contrast on delayed phase as well as positive enhancement on the subtracted images.

Focal area at the margin of the ablation zone that shows:

- Early or late arterial phase enhancement that must be proved by the subtraction images.

- Contrast wash out: the lesion becomes hypointense relative to the liver parenchyma in the delayed phase.

- This should coincide with are area of restriction on the DWI and ADC maps

\section{Statistical method and analysis}

Data were coded and entered using the statistical package SPSS (Statistical Package for the Social Science; SPSS Inc., Chicago, IL, USA) version 22. Data were summarized using mean, standard deviation, minimum and maximum in quantitative data and using frequency (count) and relative frequency (percentage) for categorical data. Standard diagnostic indices including sensitivity, specificity, positive predictive value (PPV), negative predictive value (NPV) and diagnostic efficacy were calculated. ROC curve was constructed with area under curve analysis performed to detect best cutoff value of ADC for detection of residue. For comparing categorical data, Chi square test was performed. Exact test was used instead when the expected frequency is less than 5. Agreement between the 2 readers was done using Kappa measure of agreement.

A p-value less or equal to 0.05 was considered significant and less than 0.01 was considered highly significant.

As a rule of thumb values of Kappa from 0.40 to 0.59 are considered moderate, 0.60 to 0.79 substantial, and 0.80 outstanding.

N.B: Most statisticians prefer for Kappa values to be at least 0.6 and most often higher than 0.7 before claiming a good level of agreement.

\section{RESULTS}

This was a retrospective study included 32 patients with 54 HCC lesions with ages ranging from 59 to 73 with the mean of age of 59.6 years. Of the 32 patients only 6 were females and 26 were males. HCC has a higher prevalence in the male population.

In $1^{\mathrm{ry}}$ response and follow up findings post TACE. Where good response was defined as disappearance of any intra tumoral arterial enhancement in treated lesions, residual disease was defined as at least $30 \%$ reduction in the sum of diameters of the viable enhancing lesion in the arterial phase. No response was less than 30\% reduction in the enhancing lesion diameters. Progressive disease was defined as $20 \%$ increase in the sum of diameters of the enhancing lesions in comparison to the target lesion diameter at the start of treatment.

Table (2): General evaluation of the cases according to mRECIST and EASL criteria by both readers

\begin{tabular}{|c|l|c|c|c|c|}
\hline \multirow{2}{*}{} & \multicolumn{2}{|c|}{ Reader1 } & \multicolumn{2}{c|}{ Reader2 } \\
\cline { 3 - 6 } \multicolumn{2}{|c|}{} & Count & \% & Count & \% \\
\hline \hline \multirow{3}{*}{ response } & good response & 20 & $37.0 \%$ & 18 & $33.3 \%$ \\
\cline { 2 - 6 } & RESIDUE & 34 & $63.0 \%$ & 36 & $66.7 \%$ \\
\cline { 2 - 6 } & no response & 0 & $.0 \%$ & 0 & $.0 \%$ \\
\hline \multirow{3}{*}{ Follow up } & Regression & 8 & $47.1 \%$ & 8 & $47.1 \%$ \\
\cline { 2 - 6 } & Progressive & 2 & $11.8 \%$ & 1 & $5.9 \%$ \\
\cline { 2 - 6 } & Stationary & 7 & $41.2 \%$ & 8 & $47.1 \%$ \\
\hline
\end{tabular}

Table (1) shows that residual disease was detected in $63 \%$ and $66 \%$ of the lesions by reader 1 and 2 respectively.

On the other hand $\mathbf{3 7 \%}$ and $\mathbf{3 3 . 3 \%}$ of the cases showed a good therapeutic response respectively. Both readers agreed that none of the cases showed no response to treatment. On follow up for $\mathbf{1 7}$ of those lesions regression was confirmed in $\mathbf{4 7 . 1 \%}$ of the lesions by both readers, while progressive course was detected in $\mathbf{1 1 . 8 \%}$ by reader 1 and $\mathbf{5 . 9 \%}$ by reader 2 respectively. A stationary course was observed in $\mathbf{4 1 . 2 \%}$ and $47.1 \%$ by reader 1 and reader 2 respectively. 
Table (2): Correlated the data obtained from both readers including all results of DWI, subtracted dynamic image, and THRIVE.

\begin{tabular}{|c|c|c|c|c|c|}
\hline & \multicolumn{2}{|c|}{ Reader 1} & \multicolumn{2}{|c|}{ Reader 2} \\
\hline & & Count & $\%$ & Count & $\%$ \\
\hline \multirow{2}{*}{ DWI } & FACILITATED (-ve) & 25 & $46.3 \%$ & 26 & $48.1 \%$ \\
\hline & RESTRICTED (+ve) & 29 & $53.7 \%$ & 28 & $51.9 \%$ \\
\hline \multirow{2}{*}{ subtraction } & nonenhancing / enhancing reactive & 21 & $38.9 \%$ & 21 & $38.9 \%$ \\
\hline & enhancing residue & 33 & $61.1 \%$ & 33 & $61.1 \%$ \\
\hline \multirow{2}{*}{$\begin{array}{l}\text { THRIVE } \\
\text { (dynamic) }\end{array}$} & nonenhancing / enhancing reactive & 20 & $37.0 \%$ & 20 & $37.0 \%$ \\
\hline & enhancing residue & 34 & $63.0 \%$ & 34 & $63.0 \%$ \\
\hline
\end{tabular}

Table (2) Interpreted the results for each reader obtained from all three sequences in the form of facilitated vs restricted for DWI and enhancing or not in both subtraction and THRIVE. Furthermore all results were expressed as a percentage from the total number of lesions. Although both readers agreed that 34 lesions had residual disease by conventional dynamic MRI, subtraction was able to deduct the number to 33 enhancing lesions by removing native T1 signal from enhanced images. It also increased the conspicuity of the embolized lesions for both readers achieving a higher confidence level.

In spite of under estimating the disease burden in 5 cases by reader 1 and 6 cases by reader 2, DWI still presented as a useful confirmative tool with other diagnostic series in $53.7 \%$ and $52 \%$ of lesions by reader1 and 2 respectively.

Table (3): Correlation of DWI to THRIVE noting the efficiency of DWI in detecting residual disease post TACE by reader 1

\begin{tabular}{|c|c|c|c|c|c|}
\hline & & \multicolumn{4}{|c|}{ THRIVE (dynamic) (reader 1) } \\
\hline & & \multicolumn{2}{|c|}{ Enhancing residue } & \multicolumn{2}{|c|}{$\begin{array}{c}\text { Nonenhancing / } \\
\text { enhancing reactive }\end{array}$} \\
\hline & & Count & $\%$ & Count & $\%$ \\
\hline \multirow{2}{*}{$\begin{array}{l}\text { DWI } \\
\text { (reader 1) }\end{array}$} & RESTRICTED (+ve) & 24 & 70.6\% & $\overline{5}$ & $25 \%$ \\
\hline & FACILITATED (-ve) & 10 & $29.4 \%$ & 15 & $75 \%$ \\
\hline
\end{tabular}

Table (3) illustrates how facilitated lesions on DWI represent good therapeutic of $75 \%$ of nonenhancing lesions, while around $30 \%$ of the facilitated lesions were enhancing (i.e. false negative). On the other hand $70 \%$ of the restricted lesions were enhancing on THRIVE with only $5 \%$ being restricted and nonenhancing (i.e. false positive).

False negative results were attributed to $1^{\text {ry }}$ high ADC value of well differentiated HCC, so these lesions still showed enhancement due to partial response to TACE while they were falsely facilitated on DWI.

False positive results were attributed to lipiodol deposition in the tumor giving no enhancement but only false restriction on DWI.

Table (4): Correlation of DWI to THRIVE noting the efficiency of DWI in detecting residual disease post TACE by reader 2

\begin{tabular}{|c|c|c|c|c|c|}
\hline & \multicolumn{4}{|c|}{ THRIVE (dynamic) (reader 2) } \\
\hline & & \multicolumn{2}{|c|}{ enhancing residue } & \multicolumn{2}{|c|}{$\begin{array}{c}\text { Nonenhancing / } \\
\text { enhancing reactive }\end{array}$} \\
\hline & & Count & $\%$ & Count & $\%$ \\
\hline \multirow{2}{*}{ DWI (reader 2) } & RESTRICTED (+ve) & 26 & $76.5 \%$ & 2 & $\mathbf{1 0 . 0 \%}$ \\
\hline & FACILITATED (-ve) & 8 & $23.5 \%$ & 18 & $90.0 \%$ \\
\hline
\end{tabular}

Table (4) illustrates how facilitated lesions represent good therapeutic response in up to $90 \%$ of nonenhancing lesions, while around $23 \%$ of the facilitated lesions were enhancing (i.e. false negative). On the other hand $76 \%$ of the restricted lesions were enhancing on THRIVE with only $10 \%$ being restricted and nonenhancing (i.e. false positive). 
Table (5): Accuracy measures of both DWI and subtraction as viewed by reader1

\begin{tabular}{|l|c|c|c|c||}
\hline & \multicolumn{2}{|c|}{ DWI (reader 1) } & \multicolumn{2}{c|}{ Subtraction (reader 1) } \\
\hline tatistic & Value & $\mathbf{9 5 \%}$ CI & Value & 95\% CI \\
\hline \hline ensitivity & $70.59 \%$ & $52.52 \%$ to $84.90 \%$ & $97.06 \%$ & $84.67 \%$ to $99.93 \%$ \\
\hline pecificity & $75.00 \%$ & $50.90 \%$ to $91.34 \%$ & $100.00 \%$ & $83.16 \%$ to $100.00 \%$ \\
\hline ositive Predictive value & $82.76 \%$ & $64.23 \%$ to $94.15 \%$ & $100.00 \%$ & $89.42 \%$ to $100.00 \%$ \\
\hline egative Predictive value & $60.00 \%$ & $38.67 \%$ to $78.87 \%$ & $95.24 \%$ & $76.18 \%$ to $99.88 \%$ \\
\hline
\end{tabular}

According to table (5) reader 1 subtraction dynamic MRI had a sensitivity of $97.06 \%$, a specificity of $100 \%$, a positive predictive value of $100 \%$ and a negative predictive value of $95 \%$. Compared to $70.59 \%$, $75 \%, 82.76 \%$ and $60 \%$ respectively in diffusion weighted imaging.

Table (6): Accuracy measures of both DWI and subtraction as viewed by reader 2

\begin{tabular}{|l|c|c|c|c|}
\hline & \multicolumn{2}{|c|}{ DWI (reader 2) } & \multicolumn{2}{c|}{ Subtraction (reader 2) } \\
\hline tatistic & Value & 95\% CI & Value & 95\% CI \\
\hline \hline ensitivity & $76.47 \%$ & $58.83 \%$ to $89.25 \%$ & $97.06 \%$ & $84.67 \%$ to $99.93 \%$ \\
\hline $\begin{array}{l}\text { pecificity } \\
\text { ositive Predictive } \\
\text { alue }\end{array}$ & $90.00 \%$ & $68.30 \%$ to $98.77 \%$ & $100.00 \%$ & $83.16 \%$ to $100.00 \%$ \\
\hline $\begin{array}{l}\text { Jegative Predictive } \\
\text { alue }\end{array}$ & $92.86 \%$ & $76.50 \%$ to $99.12 \%$ & $100.00 \%$ & $89.42 \%$ to $100.00 \%$ \\
\hline
\end{tabular}

According to table (5) subtraction dynamic MRI had a sensitivity of $97.06 \%$, a specificity of $100 \%$, a positive predictive value of $100 \%$ and a negative predictive value of $95 \%$. Compared to $76.47 \%, 90 \%, 92.86 \%$ and $69.23 \%$ respectively in diffusion weighted imaging.

- DWI significant agreement of (kappa=0.728) and $P$ value less than 0.001

- Subtraction significant agreement of (kappa=0.766) and $P$ value less than 0.001

- THRIVE significant agreement of (kappa=0.841) and $P$ value less than 0.001

Agreement between reader 1 and reader 2 in response (kappa $=\mathbf{0 . 8 3 8}$ ) and $\mathrm{P}$ value less than 0.001 .

In our study, the mean ADC value for necrotic lesions was 1.16 and $1.24 \times 10^{-3} \mathrm{~mm}^{2} / \mathrm{sec}$ as detected by reader 1 and 2 respectively. The mean ADC for residual disease was 1.07 and $1.05 \times 10^{-3}$ $\mathrm{mm}^{2} / \mathrm{sec}$ as detected by both readers respectively.

\section{Discussion}

Hepatocellular carcinoma (HCC) is one of the main causes of death among cirrhotic patients. Transarterial chemoembolization (TACE) is commonly employed to treat HCC patients who are not candidates for curative surgical resection or transplantation. TACE is currently recommended as first-line palliative therapy for non-surgical patients ${ }^{(\mathbf{1 0})}$.

MRI is used to differentiate completely treated zones from those containing residual tumor. MRI enhancement characteristics are an indicator for treatment efficacy and may suggest the need for further intervention. Reduction in tumor size is a validated parameter used to assess treatment response [response evaluation criteria in solid tumors (RECIST)] however, this does not take into account that non-viable HCC may show no reduction in size in the early posttreatment period (11). Tumor enhancement is used as a biomarker of disease response, as proposed by the American Association for the Study of Liver Disease (AASLD) and the European Association for the Study of the Liver (EASL) ${ }^{(11)}$. Nodular and/or irregular enhancement at the treated lesion on contrast-enhanced MRI is a sign of residual tumor. While, no enhancement or thin uniform halo enhancement (secondary to reactive hyperemia) is a sign of complete treatment necrosis ${ }^{(12)}$.

However, conventional gadoliniumenhanced MRI might not discern therapy-induced inflammation and granulation tissue from viable tumor ${ }^{(\mathbf{1 2})}$. Given these limitations, alternative MRI techniques are desired to reflect tumor response accurately ${ }^{(\mathbf{1 3})}$.

Treated HCC may show high signal on the unenhanced T1-weighted images secondary to coagulative hemorrhagic necrosis. Contrastenhanced T1-weighted high signal may be a result of enhancement, pre-existing T1-weighted high signal, or a combination of both. Subtraction MRI is a technique whereby corresponding contrastenhanced and unenhanced T1-weighted sequences are digitally subtracted using post-processing MRI software. The objective of this process is to remove pre-existing T1-weighted high signal from the post-processed images so that the remaining high signal is solely due to enhancement ${ }^{(\mathbf{1 4})}$. 
The Role of Diffusion Weighted Magnetic Resonance Imaging...

Functional MRI techniques such as diffusion-weighted imaging (DWI), detects early MR signal changes in tissues within several weeks of treatment, based on the degree of cell membrane integrity ${ }^{(\mathbf{1 5})}$. The intact membranes of viable tumor cells restrict water diffusion, whereas necrotic tumor cells with disrupted cell membranes exhibit increased water diffusion. This mobility of water is quantified by a constant known as the apparent diffusion coefficient (ADC). Therefore tumor necrosis is associated with an increase in ADC value, thereby allowing differentiation between viable and necrotic portions of tumor ${ }^{(16)}$.

The hypothesis of the present study was to improve reader confidence in differentiating residual disease from reactive hyperemia and granulation tissue thus improve the overall MRI assessment of treatment response following locoregional therapy. We aim to compare contrast enhanced subtraction MRI to functional DWI MRI in the assessment of treatment response following TACE.

\section{Interobserver agreement:}

In our study there was a high level of agreement between the two observers regarding percentage necrosis in the three phases of contrast enhancement of the subtraction images, ADC values and DWI evaluation and the overall evaluation of the cases according to RECSIST criteria:

These values were comparable to the study of Manelli et al. ${ }^{(17)}$, who had agreement values of 0.92-0.95 for subtraction imaging and 0.95 for ADC.

\section{Quantitative analysis of DWI by ADC measurement:}

It should be noted that measurements of ADC values are always subjective to some inter and intra observer variability ${ }^{(\mathbf{1 8})}$.

Intra-observer and inter observer variability were between $10 \%$ and $13.3 \%$.

We were not able to calculate an ADC cut off value from the ROC curve because the AUC was insignificant and false-negative identification of necrotic tissue may result from welldifferentiated HCC. Similar to what was found by Yuan et al. ${ }^{(15)}$, who concluded that, viable and necrotic tumor tissues may occasionally be difficult to characterize with the visual assessment of the DW-MRI alone.

Yet we could still calculate a post treatment average for good and poor responding lesions.

Similarly, Lu et al. ${ }^{(18)}$, found that viable lesions values were $0.97 \pm 0.39 \times 10^{-3} \mathrm{~mm}^{2} / \mathrm{sec}$ and non- viable lesions were $1.18 \pm 0.34 \times 10^{-3} \mathrm{~mm}^{2} / \mathrm{sec}$ $(\mathrm{p}=0.002)$
In our study of DWI, reader 1 evaluation yielded a sensitivity of $70.59 \%$, a specificity of $75 \%$, a positive predictive value of $82.76 \%$ and a negative predictive value of $60 \%$ and $95 \%$ confidence interval of (64-94\%) compared to, $76.47 \%, 90 \%, 92.86 \%$ and $69.23 \%$ and $(76-99 \%)$ respectively in reader 2 .

Our results correlated to Osama et al. ${ }^{(19)}$, who observed that diffusion weighted imaging had a sensitivity $100 \%$ of a specificity of $65.5 \%$, a positive predictive value of $67.7 \%$, a negative predictive value of $100 \%$ and an overall agreement of $80 \%$.

\section{DWI had the following causes of false negative results:}

Firstly, initial high ADC of tumor in cases of well differentiated HCC as. Secondly, Circular ROI (region of interest) used for ADC may falsely include areas of necrotic adjacent tissue. Peripheral subcapsular lesions included peritoneal fat; this may also raise the ADC values. Lastly, small subcentimetric lesions aren't clearly seen ${ }^{(15)}$.

\section{DWI causes of false positive results:}

Firstly, initial poorly differentiated HCC, these lesions should have better been assessed preand post-treatment with TACE to detect the percentage increase of ADC. This wasn't the case in our study. We only aimed to detect post TACE therapeutic response. Secondly, coagulative necrosis and hemorrhage may falsely lower ADC values. Finally, intralesional lipidol accumulation may lead to intralesional restriction.

Kamel et al. ${ }^{(16)}$ and Yuan et al. ${ }^{(15)}$, reported that DWI and ADC values quantify tumor necrosis following chemoembolization to a greater degree than gadolinium-enhanced MRI. In addition, Kamel et al. ${ }^{(\mathbf{1 6})}$ found that ADC values exhibited a higher correlation with the degree of tumor necrosis at pathology $(\mathrm{P}<0.05)$ than on gadoliniumenhanced MRI. However Yuan et al. ${ }^{(15)}$ didn't have any form of histological correlation. On the other hand Kamel et al. ${ }^{(16)}$, included only a small number of patients and didn't assess image subtraction or report the details of acquisition and processing of the histopathologic specimens which were limiting factors of his study.

However, our results agreed with Mannelli $\boldsymbol{e t}$ al. (17) who showed that compared to contrastenhanced MRI, subtraction technique exhibited a more significant correlation with the histopathological findings than DWI. In addition, Goshima et al. ${ }^{(20)}$ stated that DWI was not a reliable predictor of local HCC recurrence following TACE when compared with gadolinium-enhanced MRI.

However in particular, the degree of tumor necrosis of large HCC following TACE may be predicted by DWI, and patient management may be 
guided by the results. The ADC value may also be used to predict the survival of patients with HCC following TACE ${ }^{(\mathbf{1 5})}$.

In conclusion, DWI can quantify HCC tumor necrosis following chemoembolization, and the ADC value may be useful to determine necrotic and viable tumor tissues. Additionally, DW-MRI shows improved liver lesion detection. Therefore, DWI may be an option for the short-term follow-up of HCC patients following chemoembolization and may guide patient management for reducing radiation exposure of $\mathrm{CT}$ examination and the risk of contrast material-induced nephropathy ${ }^{(15)}$.

\section{Image subtraction:}

In our study we noted that both readers had the same statistical values as noted; sensitivity $97.06 \%$ specificity $\% 100$, positive predictive value $100 \%$, NPV $95.24 \%$. Consequently both readers agreed to a PPV of $100 \%$ with CI $95 \%=89-100 \%$, NPV of $95 \%$ with CI $95 \%=76-99 \%$.Our results correlated to Bolog et al. ${ }^{(21)}$ _who reported a sensitivity of $92 \%$, specificity of $100 \%$, and accuracy of $93 \%$ for the detection of viable tumor after TACE (in $30 \mathrm{HCCs}$ ) using double-contrast MRI [superparamagnetic iron oxide (SPIO) and Gd-DTPA agents].

Being able to bridge the previously described DWI pitfalls, subtraction gained higher sensitivity and specificity and confidence interval by:

Firstly, providing specific contrast enhancement patterns as described by Winters et al. ${ }^{22}$.

- Peripheral nodular enhancement for residual disease,

- Rim contrast enhancement for reactive perilesional hyperemia

- No enhancement for well ablated lesions.

Secondly, small sized tumors were challenging yet diagnosis was still amenable as detected in our study.

Many of the lesions ranged in size from (0.8$1.5 \mathrm{~cm}$ ) were clearly depicted on subtraction but not seen on DWI.

However, Winters et al. ${ }^{(22)}$,excluded all lesions below $1 \mathrm{~cm}$ in size from his study in fear that they may be vulnerable to image misregistration ${ }^{(23)}$.

Thirdly, motion artefacts weren't as of such great effect on the diagnostic series especially in cases with minimal misregistration artefacts, the confidence interval was still high. This was consistent to Kim et al. ${ }^{(24)}$ in their study, there were eight cases of minimal misregistration. The correlation between image subtraction and pathologic assessment of tumor necrosis was slightly lower in misregistered cases, but still remained significant.

\section{Limitations of the study were as follows:}

This was a retrospective study so it is prone to the limitations inherent to all studies of this design.

The sample size was small due to the strict selection criteria adopted for this highly specialized indication. This reduced the power of the statistical analysis. Kim et al. ${ }^{(24)}$ found a significant correlation between subtraction MRI and the histopathological assessment of $\mathrm{TZ}$ necrosis with significantly higher sensitivity and accuracy for the diagnosis of complete necrosis compared to standard MRI. Mannelli et al. ${ }^{(17)}$ found a significant correlation between subtraction MRI and the pathological percentage of $\mathrm{TZ}$ necrosis. Despite the small sample size in the present study, a statistically significant ( $\mathrm{p}=0.001$ and 0.001 ) superior reader confidence level was proven for the subtraction MRI protocol versus the standard MRI protocol. This was an important finding not previously published in the literature for this specific study population.

The SOR was not based on histology. No biopsies were performed to confirm complete treatment necrosis or residual disease; due to ethical and practical difficulties in performing $\mathrm{TZ}$ biopsies in all cases. Aside from procedural invasiveness, performing $\mathrm{TZ}$ biopsies is associated with technical difficulties in obtaining adequate tissue sampling. False-negative results are not uncommon as foci of residual disease are often small targets and difficult to discriminate on image-guided biopsy. In fact, the only true reference standard is the liver explant. This will be done only if our patients are scheduled for hepatic transplantation.

We did not assess DWI as a standalone sequence in the diagnosis of tumor necrosis after TACE. Instead, image subtraction was used to define necrosis on images, and this decision may have introduced bias. It would be interesting to perform future studies comparing DWI with contrast-enhanced imaging in a more independent manner.

We included the cases of minimal misregistration of image subtraction in our study. Subtraction technique helps increased depiction of enhancement that are hyperintense on unenhanced images, misregistration is a disadvantage of subtraction that results in either spurious peripheral enhancement or signal loss. However, misregistration artefacts in our cases were minimal, and according to Kim et al. ${ }^{(24)}$ the correlation of image subtraction and pathological assessment of tumor necrosis remained significant in minimally misregistered cases. 


\section{CONCLUSION AND RECOMMENDATIONS}

MRI is a powerful tool in detection of residual tumor viability, quantifying tumor necrosis and detecting complications after TACE. Imaging protocol should include dynamic study combined with post processing subtraction images for better tissue characterization. Compared to DWI, subtraction MRI is much more valuable, where it increases radiologists' confidence in interpreting treatment response following loco-regional therapies for HCC. DWI can still provide acceptable values of accuracy and can be used for patients who can't receive GD-DTPA and to quantify tumor necrosis in large HCC. This may help to facilitate the appropriate clinical management of patients including the need for re-treatment.

\section{REFERENCES}

1) Jemal A, Bray $F$ and Center $M$ et al. (2011): Global cancer statistics. CA Cancer J Clin., 61:69-90.

2) Llovet J, Real M, Montaña X et al. (2002): Arterial embolization or chemoembolisation versus symptomatic treatment in patients with unresectable hepatocellular carcinoma: a randomized controlled trial. Lancet, 359:1734-1739.

3) Befeler A and Di Bisceglie A (2002): Hepatocellular carcinoma: diagnosis and treatment. Gastroenterology, 122:1609-1619.

4) Braga L, Guller U and Semelka R (2005): Pre-, peri-, and post-treatment imaging of liver lesions. RadiolClin North Am., 43:915-927.

5) Lee $C$, Bragac $L$, de Campos $R$ and Semelka $R$ (2011): Hepatic tumor response evaluation by MRI.NMR Biomed., 24(6):721-33.

6) Riaz A, Miller F, Kulik L et al. (2010): Imaging Response in the Primary Index Lesion and Clinical Outcomes Following TransarterialLocoregional Therapy for Hepatocellular Carcinoma. JAMA., 303: 1062-1069.

7) Yuan Z, Ye X, Dong S et al. (2010): Role of magnetic resonance diffusion-weighted imaging in evaluating response after chemoembolization of hepatocellular carcinoma. Eur J Radiol., 75: 9-14.

8) Newatia A, Khatri G and Friedman B et al. (2007): Subtraction Imaging: Applications for Nonvascular Abdominal MRI. AJR., 188:1018-1025.

9) Chopra S, Dodd GD, Chintapalli KN, Leyendecker JR, Karahan OI and Rhim H (2001): Tumor recurrence after radiofrequency thermal ablation of hepatic tumors: spectrum of findings on dual-phase contrast-enhanced CT. AJR Am J Roentgenol., 177:381-387.

10) Özkavukcu E, Haliloğlu N and Erden A (2009): Post-treatment MRI findings of hepatocellular carcinoma. DiagnInterv Radiol., 15:111-120.
11) Kamel I and Morgan $H$ (2011): MRI Appearance of Treated Liver Lesions. nProc Intl Soc Mag Reson Med., (19): $1-6$

12) Lim H and Han J (2002): Hepatocellular carcinoma: evaluation of therapeutic response to interventional procedures. Abdom Imaging, 27:168-179.

13) Chen C, Li C, Kuo Y, Jaw T, Wu D et al. (2006): Early response of hepatocellular carcinoma to transcatheter arterial chemoembolization: choline levels and MR diffusion constants--initial experience. Radiology, 239:448-456.

14) Friedman $B$ and Hines $J$ et al. (2007): Subtraction nonvascular applications. Research gate, 188: 10181025.

15) Yuan Z, Li W, Ye X, Peng W, Xiao $X$ (2014): Utility of diffusionweighted imaging to assess hepatocellular carcinoma viability following transarterial chemoembolization, 222: 831-836.

16) Kamel I, Bluemke D, Ramsey D et al. (2003): Role of diffusion-weighted imaging in estimating tumor necrosis after chemoembolization of hepatocellular carcinoma. AJR., 181:708-710.

17) Mannelli L, Kim S, Hajdu $C$ et al. (2009): Assessment of tumor necrosis of hepatocellular carcinoma after chemoembolization: diffusion-weighted and contrast-enhanced MRI with histopathologic correlation of the explanted liver. Am. J. Roentgenol., 193: 1044-1052.

18) Lu TL, Meuli RA, Marques-Vidal PM, Bize P, Denys A and Schmidt S (2010): Interobserver and intraobserver variability of the apparent diffusion coefficient in treated malignant hepatic lesions on a 3.0 $\mathrm{T}$ machine: measurements in the whole lesion versus in the area with the most restricted diffusion. Journal of Magnetic Resonance Imaging, 32(3):647-53.

19)Osama R, Abdelmaksoud A, El Tatawy S, Mohammed Nabeel M et al. (2013): Role of dynamic contrast-enhanced and diffusion weighted MRI in evaluation of necrosis of hepatocellular carcinoma after chemoembolization. Egyptian Journal of radiology and nuclear medicine, 44: 737-746.

20) Goshima S, Kanematsu M, Kondo $H$ et al. (2008): Evaluating local hepatocellular carcinoma recurrence post-transcatheter arterial chemoembolization: is diffusion-weighted MRI reliable as an indicator? J MagnReson Imaging, 27:834-839.

21)Bolog N, Pfammatter T, Mullhaupt B et al. ( 2008) Yu J and Rofsky N (2003): Dynamic Subtraction MR Imaging of the Liver: Advantages and Pitfalls. AJR, 180:1351-1357.

22)Winters S, Jackson S, Armstrong G et al. (2012): Value of subtraction MRI in assessing treatment response following image-guided loco-regional therapies for hepatocellular carcinoma. Clinical Radiology, 67 : 649-655.

24)Kim S, Mannelli L, Hajdu C et al. (2010): Hepatocellular carcinoma: assessment of response to transarterial chemoembolization with image subtraction. J MagnReson Imaging, 31: 348-355. 